\title{
An Integrated Approach for Process Control Valves Diagnosis Using Fuzzy Logic
}

\author{
Alvaro Luiz G. Carneiro1, Almir C. S. Porto Jr. ${ }^{2}$ \\ ${ }^{1}$ Nuclear Energy Research Institute/National Nuclear Energy Commission (IPEN/CNEN-SP), São Paulo, Brasil \\ ${ }^{2}$ Navy Technology Center/Navy Ministry (CTMSP/MB), São Paulo, Brasil \\ Email: carneiro@ipen.br, almir@ctmsp.mar.mil.br
}

Received 15 May 2014; revised 19 June 2014; accepted 2 July 2014

Copyright (C) 2014 by authors and Scientific Research Publishing Inc.

This work is licensed under the Creative Commons Attribution International License (CC BY). http://creativecommons.org/licenses/by/4.0/

(c) (i) Open Access

\begin{abstract}
Control valves are widely used in industry to control fluid flow in several applications. In nuclear power systems they are crucial for the safe operation of plants. Therefore, the necessity of improvements in monitoring and diagnosis methods started to be of extreme relevance, establishing as main goal of the reliability and readiness of the system components. The main focus of this work is to study the development of a model of non-intrusive monitoring and diagnosis applied to process control valves using artificial intelligence by fuzzy logic technique, contributing to the development of predictive methodologies identifying faults in incipient state. Specially in nuclear power plants, the predictive maintenance contributes to the security factor in order to diagnose in advance the occurrence of a possible failure, preventing severs situations. The control valve analyzed belongs to a steam plant which simulates the secondary circuit of a PWR-Pressurized Water Reactor. The maintenance programs are being implemented based on the ability to diagnose modes of degradation and to take measures to prevent incipient failures, improving plant reliability and reducing maintenance costs. The approach described in this paper represents an alternative departure from the conventional qualitative techniques of system analysis. The methodology used in this project is based on signatures analysis, considering the pressure (psi) in the actuator and the stem displacement $(\mathrm{mm})$ of the valve. Once the measurements baseline of the control valve is taken, it is possible to detect long-term deviations during valve lifetime, detecting in advance valve failures. This study makes use of MATLAB language through the "fuzzy logic toolbox" which uses the method of inference "Mamdani", acting by fuzzy conjunction, through Triangular Norms (t-norm) and Triangular Conorms (t-conorm). The main goal is to obtain more detailed information contained in the measured data, correlating them to failure situations in the incipient stage.
\end{abstract}

\section{Keywords}

Process Control Valve, Condition Monitoring, Diagnosis System, Fuzzy Logic 


\section{Introduction}

Monitoring, maintenance and reliability of plant equipment are important operational issues and the condition monitoring strategy in recent years has been to continue with deployment of maintenance optimization approach in the identification of malfunctions in plant components [1] [2]. This paper presents a nonintrusive diagnostic technique to identify operational malfunctions in control valves used in nuclear power plant, through the analysis of the signature of the valve considering the displacement stem (milimeters) and the air pressure (psi) variation on the actuator. The methodology used is based on the signatures analysis during the closing and opening stroke. Once the measurement baseline of the valve is taken, it is possible to detect long-term deviations during valve operation, identifying in advance valve failures. The early detection allows avoiding catastrophic failures and the associated costs, besides to prevent interference with normal operation of plant [3] [4]. This work makes use of the Fuzzy Logic, by the tool "Fuzzy-Logic Tool Box-MATLAB", that is a powerful mathematical application software of problem's solutions and graphical illustrations. The output of the diagnosis system provides the status pointing some typical malfunctions if it is the case. The diagnostic system has been tested using data from the laboratory tests and the case study presented is of a temperature control valve.

\section{Materials and Methods}

\subsection{Materials}

The case study presented on this article it is a control valve that charges combustible to the steam generator simulating a secondary circuit of a power water reactor of the laboratory of testes of propulsion equipment of the Brasilian Navy Technology Center.

The data was acquired by the Valvelink software through the electronic positioner, Figure 1 shows the valve and the intelligent positioner.

\subsection{Methods}

\section{Fuzzy Logic and Expert System}

Fuzzy Logic is a formal approach to map a set of "INPUTS" to a set of “OUTPUTS”, providing a systematic approach for making decisions which can be quantified in the form of a crisp value [5]. The main difference between classical logic and Fuzzy Logic is that if/then rules are supposed to be true or false, and in fuzzy logic they can be true to a certain degree [6].

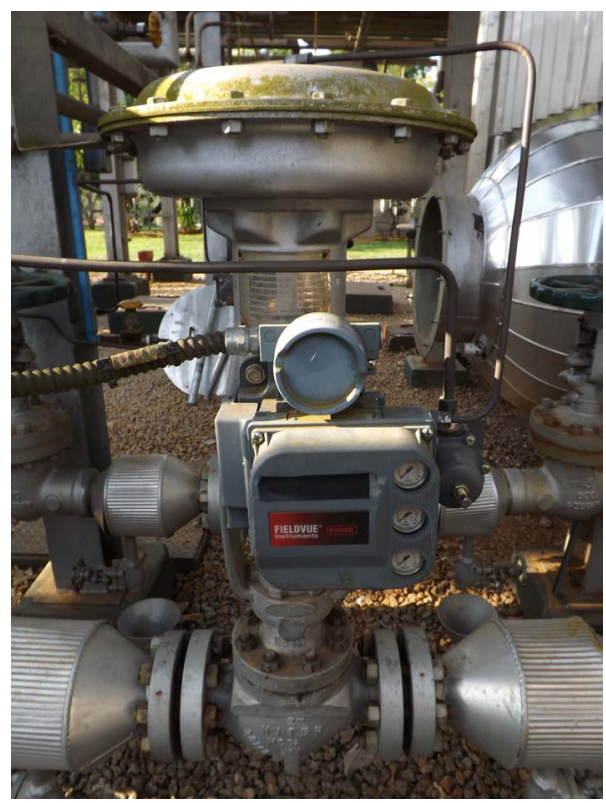

Figure 1. Control valve and the inteligente positioner. 
Along with Fuzzy Logic, expert Syetm is a branch of Artificial Intelligence, in order to solve problems at the level of a human expert. Expert systems make extensive use of specialized knowledge.

Data input and output of expert system are linguistic variables. A linguistic variable is a variable whose arguments are referred to as fuzzy values. In practice, input to the system must be fuzzified. The process of diagnosis is then performed on the fuzzy variables. After the inference procedure, the diagnosis is also expressed in fuzzy terms. Because of this, the output should be defuzzified each time a diagnosis is made. The fuzzification and defuzzification are important steps in the expert system. Figure 2 shows a block diagram of a Fuzzy Logic System (FLS).

The FLS is divided: Fuzification, Rules’ base, Inference Engine and Defuzification [7].

1) Fuzzification: is based on the concept of fuzzy set. Unlike the classical theory of sets and its mathematical logic rigidity, the Fuzzy Logic presents the term membership as a degree with gradual variations. In the Fuzzy Logic Toolbox, the input is always a crisp numerical value limited to the universe of discourse of the input variable and the output is a fuzzy degree of membership in the qualifying linguistic set (always the interval between 0 and 1 ).

Classic set $A=\{0,1\}$ discrete values 0 and 1 ; and Fuzzy Set $B=[0,1]$.

The degree of membership can assume continuous values from 0 to 1 , then the membership " $x$ " of a subset fuzzy " $B$ " of " $X$ " can be described as: $\mu B: x[0,1]$; where $\mu B$ is the degree of membership. Therefore, the fuzzification that is the first step, consists to take the inputs and determine the degree to which they belong to each of the appropriate fuzzy sets via membership functions. The membership function is a curve that defines how each point in the input space is mapped to a membership value (or degree of membership) between 0 and 1 .

The membership function can assume various geometric shapes (trapezoidal, triangular, Gaussian, etc). The fuzzy set [4] can be described as: $B=\{(x, \mu(B)(x)) \mid x \in X\}$. Each pair $(x, \mu B(X))$, that is called a singleton with a degree of membership $\mu B(x)$ in a universe of discourse.

Figure 3 presents the fuzzification step considering an input variable and a triangular membership function.

The main operations between fuzzy sets [8] are union, intersection, and complement.

Figure 4 shows the operations as follow:

Union $\mu(A \cup B)(x)=\mu A(x) \cup \mu B(x)$;

Intersection $\mu(A \cap B)(x)=\mu A(x) \cap \mu B(x)$; and

Complement $\mu A \sim(x)=1-\mu A(x)$ Complement $\mu A \sim(x)=1-\mu A(x)$.

\section{2) Rule's Base:}

In artificial intelligence, there are several ways to represent knowledge. However, one of the most common ways of representing human knowledge is through natural language expressions. Hence we have expressions like:

IF premise (antecedent), THEN conclusion (consequent).

Timothy [9] states that this representation is generally referred to as deductive way. Typically expressed an inference that is known (premise, antecedent, hypothesis), which can be inferred, or derive from, the other fact called completion (consequent). This knowledge representation called superficial knowledge is appropriate in linguistic context, because it expresses the empirical knowledge and heuristics of own communication language of the human being, capturing, so a deep form of knowledge.

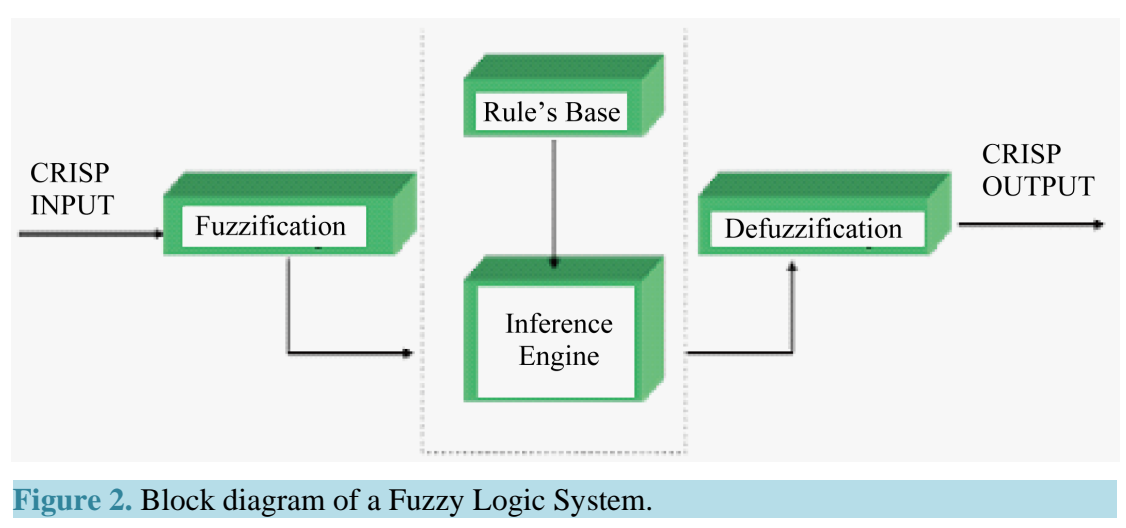




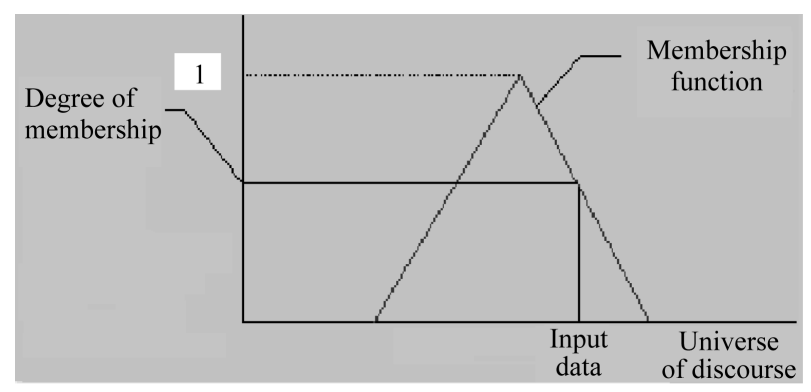

Figure 3. Fuzzification of an input variable [5].

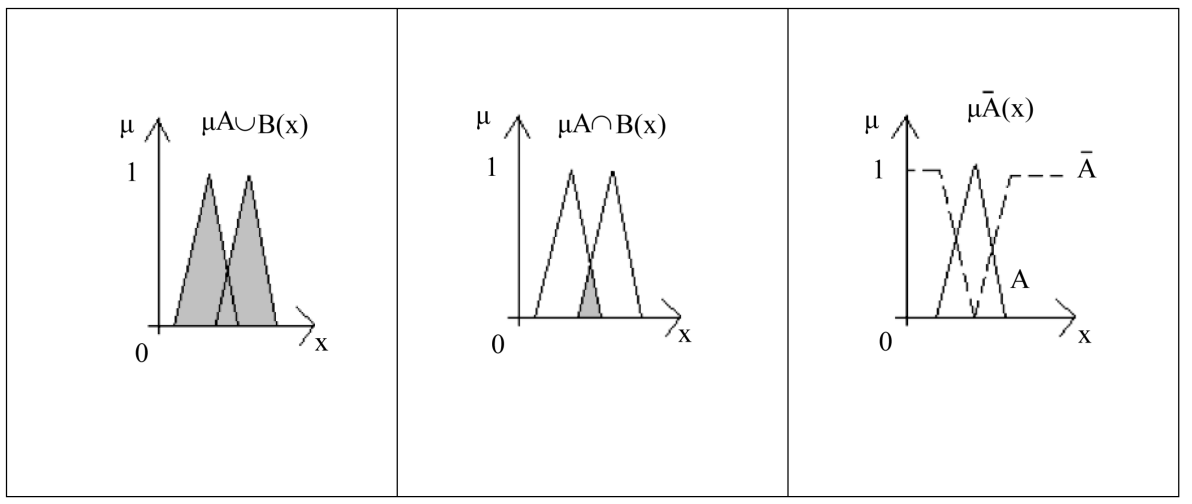

Figure 4. Union, intersection and complement of fuzzy sets.

The rules are provided by experts or can be extracted from numerical data (where the expertise of specialist in valves and the mass of data to be reported by the instrumentation team process plant). What is described by the human being (specialist) is taken as the basis for developing the rules. Each rule is part of a conditional structure with one or more clauses, such as "If A then B else C".

The rules can be classified as multi-antecedent or multi-consequent, incomplete, mixed, sentence, quantifier phrases, comparative, etc.

3) Inference Engine: is an algorithm that deals with the rules that represent the knowledge of an expert, that is a procedure used to assess the fuzzy linguistic descriptions. These procedures can be implemented on a computer when it's necessary quick processing. However, sometimes, it is useful to make an inference manually, with few rules, to check computer programs and/or to check the perfect functioning of the own inference. Several methods of inference could be cited. The most known are Systems of Mamdani [10] and Sugeno.

Considering a simple system of two rules, where each rule has two antecedents and a consequent, and that can be easily extended to fuzzy rule base or fuzzy systems with any number of antecedents and consequents. A fuzzy system with two non-interactive entries $x_{1}$ and $x_{2}$ (antecedents) and a simple output $y$ (consequent) is described by a collection of linguistic expressions "IF-THEN" proposition in the form of Mamdani:

IF $x_{1}$ is $\left(A_{1}\right) k$ and $x_{2}$ is $\left(A_{2}\right) k$ THEN $y k$ is $B k$ for $k=1,2, \ldots, r$

where $\left(A_{1}\right) k$ and $\left(A_{2}\right) k$ are fuzzy sets representing the $k$ th antecedent pair and $B k$ is the fuzzy set representing the $k$ th consequent.

It is possible to illustrate the above equation, whereas two inputs $x_{1}$ and $x_{2}$ with scalar values (crisp), with delta functions and using the max-min inference method. The member function for the inputs $x_{1}$ and $x_{2}$ are described as:

$$
\begin{gathered}
\mu\left(x_{1}\right)=\delta\left(x_{1}-\operatorname{input}(i)\right)=1, x_{1}=\operatorname{input}(i) ; 0, \\
\mu\left(x_{2}\right)=\delta\left(x_{2}-\operatorname{input}(j)\right)=1-x_{2}=\operatorname{input}(j) ; 0,
\end{gathered}
$$

Based on inference method of Mamdani implication, the output produced for $r$ rules will be as showed in Figure 5, which in this case is used the method of minimum maximum: 

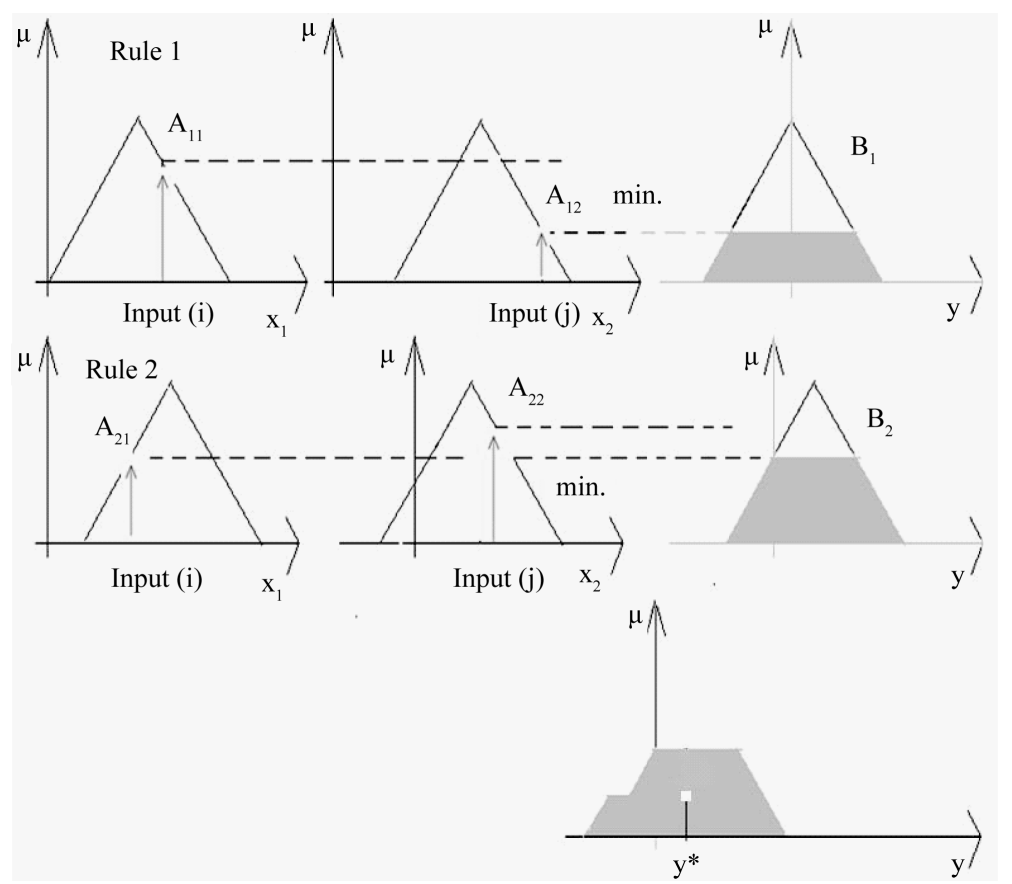

Figure 5. Example of Fuzzy Logic System with all steps.

$$
\mu(B) k(y)=\operatorname{Maxk}\left[\min \left[\mu\left(A_{1}\right) k(\operatorname{input}(i)), \mu\left(A_{2}\right) k(\text { input }(j))\right]\right], k=1,2, \cdots, r
$$

It's still possible to get the MAMDANI inference through intersection operators:

$$
R_{c}=A X B=\int U X V(\mu A(u) \cap \mu B(v)) /(u, v)
$$

4) Defuzzification: it is the conversion of a fuzzy quantity for a precise amount (crisp), while fuzzification is the reverse process. As much as fuzziness helps the rule evaluation during the intermediate steps, the final desired output for each variable is generally a single number. The output of a fuzzy process can be a logical union of two or more functions defined in the universe of discourse of an output variable. For example, a fuzzy output consists of two parts, a triangular and trapezoidal shape as shown in Figure 6.

The two top graphs in Figure 7 with member functions of trapezoidal and triangular shape, represent the two parts of fuzzy output that enveloped, involves, in this case, the union of the previous areas.

$$
\begin{gathered}
C k=U k \\
i=1 C i=C
\end{gathered}
$$

This method presents the characteristic to involve all members through the union of their areas.

The output of the FLS or specialist system is not fuzzy anymore, it is a numeric value. There are several methods used to perform defuzzification: height, height modified, maximum average, maximum, and centroid.

The centroid or center of gravity, finds the point where a vertical line would slice the aggregate set into two equal masses. Mathematically this center of gravity can be expressed as:

$$
U=\frac{\int_{\min }^{\max } u \varphi_{B}(u) \mathrm{d} u}{\int_{\min }^{\max } \varphi_{B}(u) \mathrm{d} u}
$$

The centroid method has been used on this project due to be the most appropriate method for obtaining crisp values which represent uncertain data, graphically shown in Figure 7.

\section{Results and Discussion}

The case study presented deals with the analysis and diagnosis of the temperature control valve TV-462B that 


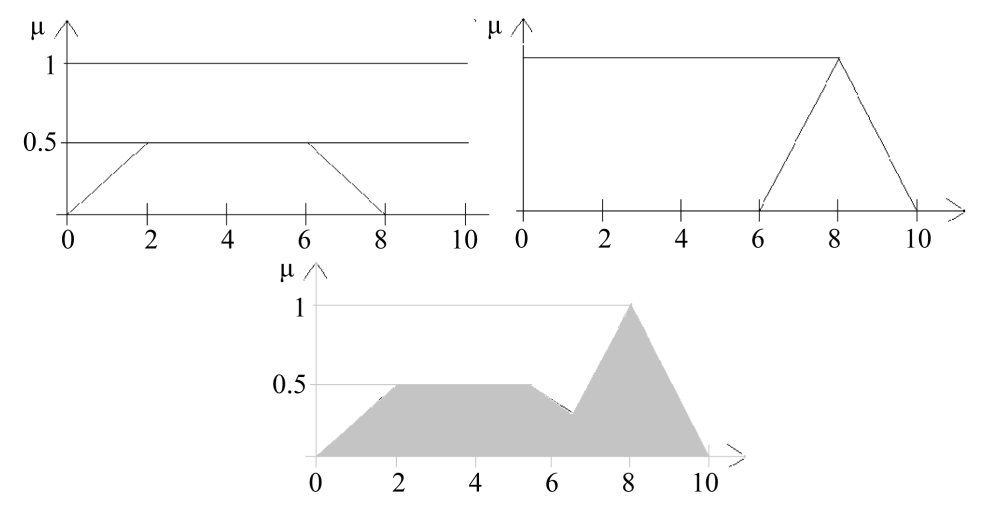

Figure 6. Typical fuzzy process output.

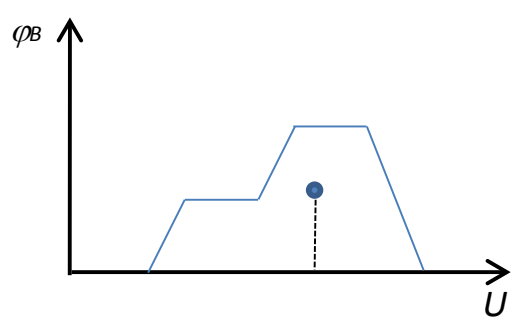

Figure 7. Center of gravity method.

performs the cooling of lubricating oil of the main propulsion turbine of the secondary circuit of a PWR. It is installed in a plant testing propulsion in the laboratory—LATEP—of the Brazilian's Navy.

There were used as input variables of the Fuzzy System, the compressed air pressure from valve's actuator, in PSI (Pressure Square Inch) and the displacement of the valve stem in mm (milimeters). The system output variable has the "status" of the valve, by percentage (0 to 100\%) of opening/closing, with indications that the valve is stuck open, stuck closed, with friction, with difficulty to open, or needing to schedule maintenance. The data (signature of the valve) acquired by the DeltaV ${ }^{\mathrm{TM}}$ automation system installed in the laboratory consist of input data of the Fuzzy Logic System-FLS.

The valve has the following features:

\begin{tabular}{cc}
\hline Input variable & Output variable \\
\hline $\begin{array}{c}\text { excvalv } \rightarrow 4 \text { to } 23 \mathrm{~mm} \\
\text { pressat } \rightarrow 0 \text { to } 28 \text { PSI }\end{array}$ & statvalv $\rightarrow 0$ to $100 \%$ (from opening to closing) \\
\hline
\end{tabular}

Figure 8 shows the correct signature (no failures) of the valve "baseline", where at the top is the closing cycle and at the bottom the opening cycle, which represents the tour of the valve stem $4-23 \mathrm{~mm}$ and 0 to 28 PSI the pressure on its diaphragm.

Table 1 shows the values of the variables and the respective valve status as output variable. The "status" closed represents that the stem touched the base. And closed strongly represents the excess air pressure to ensure that the valve is sealed to avoid leakage. The diagnosis due to the status of the valve is represented by Table 2. It shows when the stem is working normally, or is locked, or with motion difficulties or need maintenance.

Table 3 shows the membership functions and input/output variables.

Figure 9 shows an anomaly on the left in the upper cycle (close). This anomaly indicates difficulty in movement, and is transferred to the inputs of the FLS through Valvelink ${ }^{\circledR}$, module of Delta $\mathrm{V}^{\circledR}$ automation system. The data are treated according to the rule base (Table 4) and will provide an output, which in this case will then automatically diagnose as an incipient failure.

Figures 10-12 present the MATLAB ${ }^{\circledR}$ chart of membership functions of inputs “excvalv" and "pressat", and the output "statvalv" respectively.

Figure 13 shows the rules' viewer with emphasis on the 8, which the intensity of the two inputs gene- 


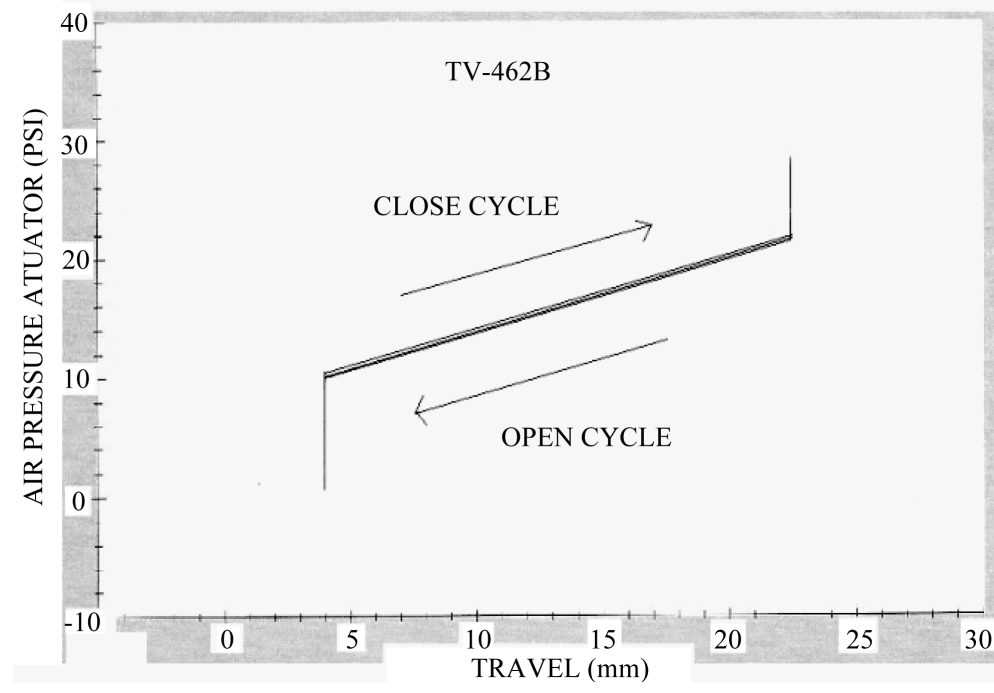

Figure 8. Valve's baseline TV-462B.

Table 1. Valve’s range.

\begin{tabular}{ccccccc}
\hline Valve Status & Open & $\mathbf{1 / 4}$ & $\mathbf{1 / 2}$ & $\mathbf{3 / 4}$ & Closed & Strongly closed \\
\hline TV462B & $(4 ; 0)$ & $(9 ; 12.5)$ & $(14 ; 15)$ & $(19 ; 17.5)$ & $(23 ; 20)$ & $(23 ; 20)(23 ; 28)$ \\
\hline
\end{tabular}

Table 2. Diagnosis by "Status" of the valve.

\begin{tabular}{ccccccc}
\hline Valve status & Stuck open & Closing normally & Opening normally & Difficulty to close & $\begin{array}{c}\text { Requiring } \\
\text { maintenance }\end{array}$ & Stuck closed \\
\hline TV462B & $(4.28)$ & $(4.0)(23.28)$ & $(23.28)(23.20)$ & $(4.12)(5.12)$ & $(20.17)(20.1)$ & $(20.0)$ \\
\hline
\end{tabular}

Table 3. The membership functions.

\begin{tabular}{|c|c|c|c|c|}
\hline TV462B & Values (excvalv) & Values (pressat) & Values (statvalv) & Membership Function \\
\hline Open & {$\left[\begin{array}{lll}3 & 4 & 7\end{array}\right]$} & {$\left[\begin{array}{lll}0 & 12 & 10\end{array}\right]$} & {$\left[\begin{array}{lll}-1 & 15 & 0\end{array}\right]$} & Trimf \\
\hline $1 / 4$ & [12 9 6 6 ] & [11 12.75 14.5] & {$\left[\begin{array}{lll}10 & 25 & 40\end{array}\right]$} & Trimf \\
\hline Half open & [11 14 17] & [15.5 14 17] & {$\left[\begin{array}{llll}30 & 50 & 70\end{array}\right]$} & Trimf \\
\hline $3 / 4$ & {$\left[\begin{array}{lll}16 & 19 & 22\end{array}\right]$} & [15.75 17 19] & [60 75 90] & Trimf \\
\hline Closed & {$\left[\begin{array}{lll}21 & 23 & 26\end{array}\right]$} & {$\left[\begin{array}{lll}18 & 21 & 24\end{array}\right]$} & [85 100 105] & Trimf \\
\hline Strongly closed & Not applicable & {$\left[\begin{array}{llll}21 & 30 & 19 & 32\end{array}\right]$} & {$\left[\begin{array}{lllll}88 & 100 & 120 & 125\end{array}\right]$} & Tramf \\
\hline Stuck open & & & {$\left[\begin{array}{lll}-3 & 0 & 5\end{array}\right]$} & Trimf \\
\hline Difficulty to close & & & {$\left[\begin{array}{lll}3 & 10 & 15\end{array}\right]$} & Trimf \\
\hline Requiring maintenance & & & {$\left[\begin{array}{llll}75 & 80 & 85\end{array}\right]$} & Trimf \\
\hline Stuck closed & & & [95 100 120] & Trimf \\
\hline
\end{tabular}

rates a diagnosis, showing a type of failure. Due the entries “excvalv” and "pressat” present as “open” and "a quarter", the output variable "statvalv” present itself with the diagnosis “difficult to close”, demonstrating an incipient failure, at the beginning of the closing valve.

\section{Conclusions}

The paper shows that expert system technology has brought significant improvements on predictive/proactive maintenance, considering the specific case, using Artificial Intelligence by the Fuzzy Logic Technique, detecting failures in advance which is a relevant contribution to condition monitoring and diagnose system. 


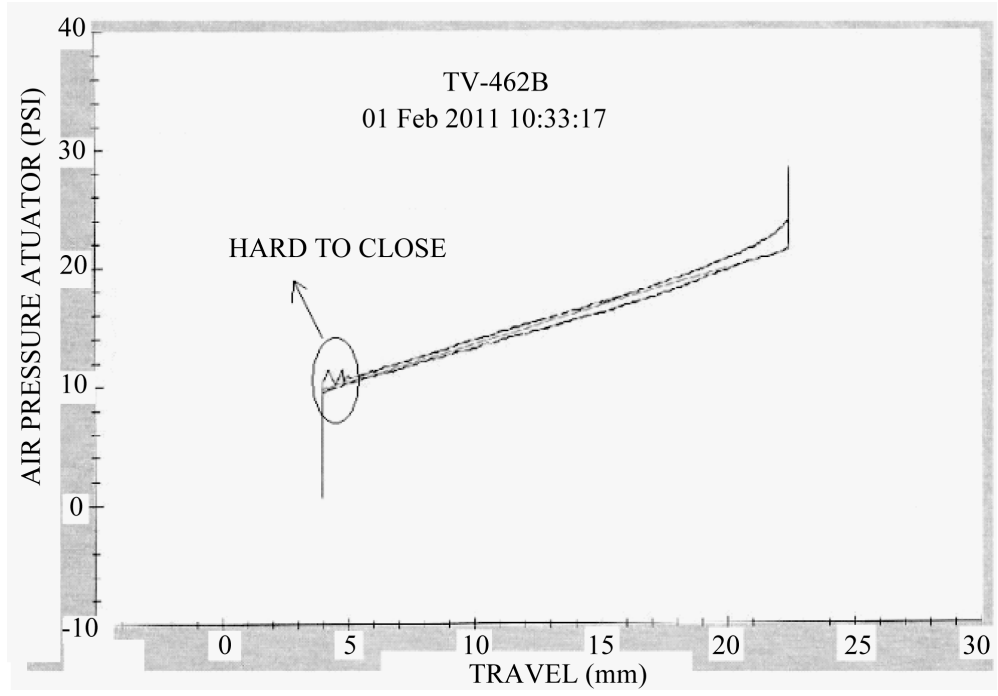

Figure 9. Valve’s signature TV-462B.

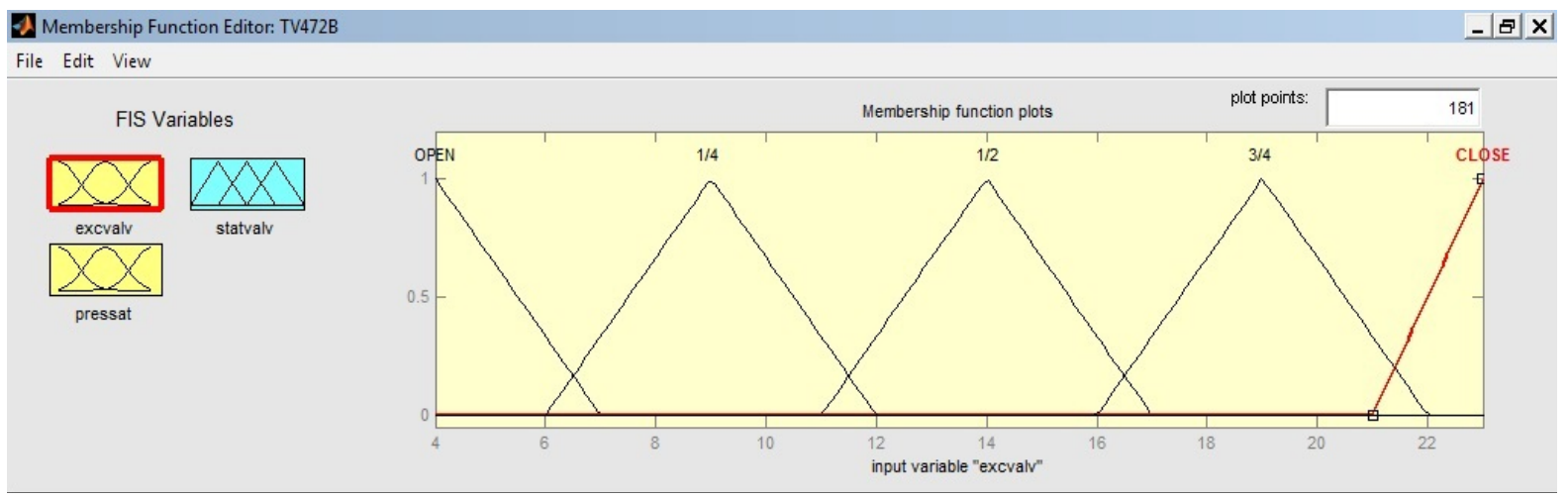

Figure 10. The input variable "excvalv".

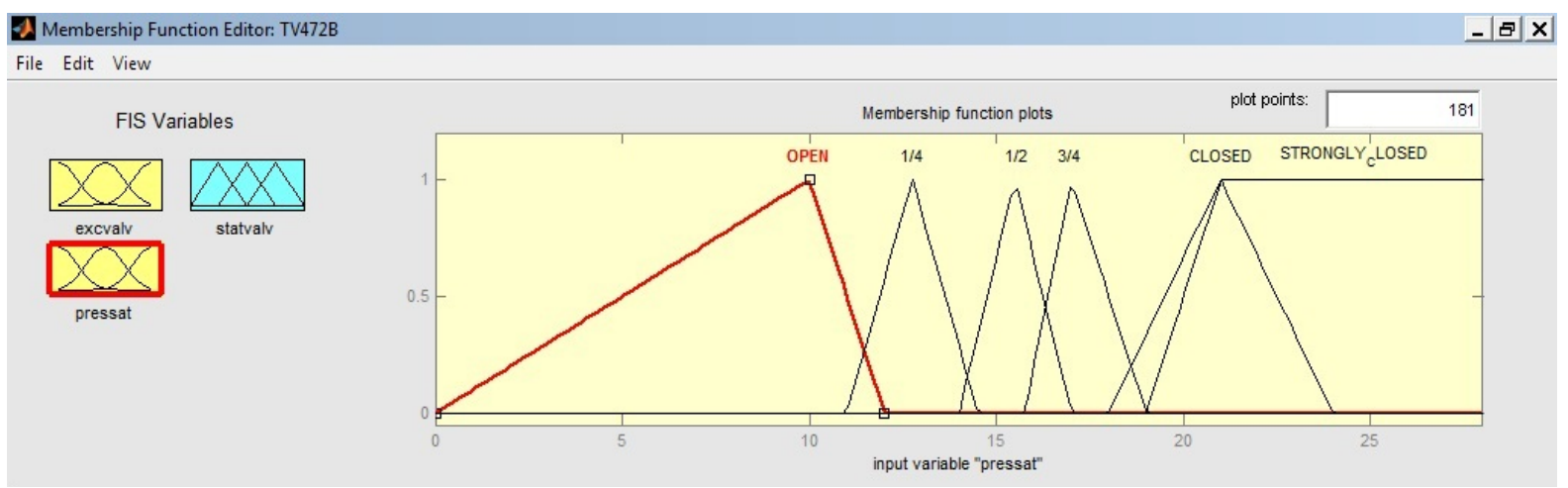

Figure 11. The input variable "pressat”.

The use of the MATLAB ${ }^{\circledR}$ platform aggregated to the Fuzzylogic toolbox has proved to be a powerful tool, using the tacit knowledge of the experts allowing the expert systems to create a favorable scenario, becoming possible to learn about the correct diagnosis of a final control element (valves) of great importance to control in industrial plant, and particularly in nuclear plant, where the availability of the equipment should be high and unexpected intervention should be avoided.

However, this technique should be improved, testing different situations of failures in order to check the per- 
Membership Function Editor: TV472B

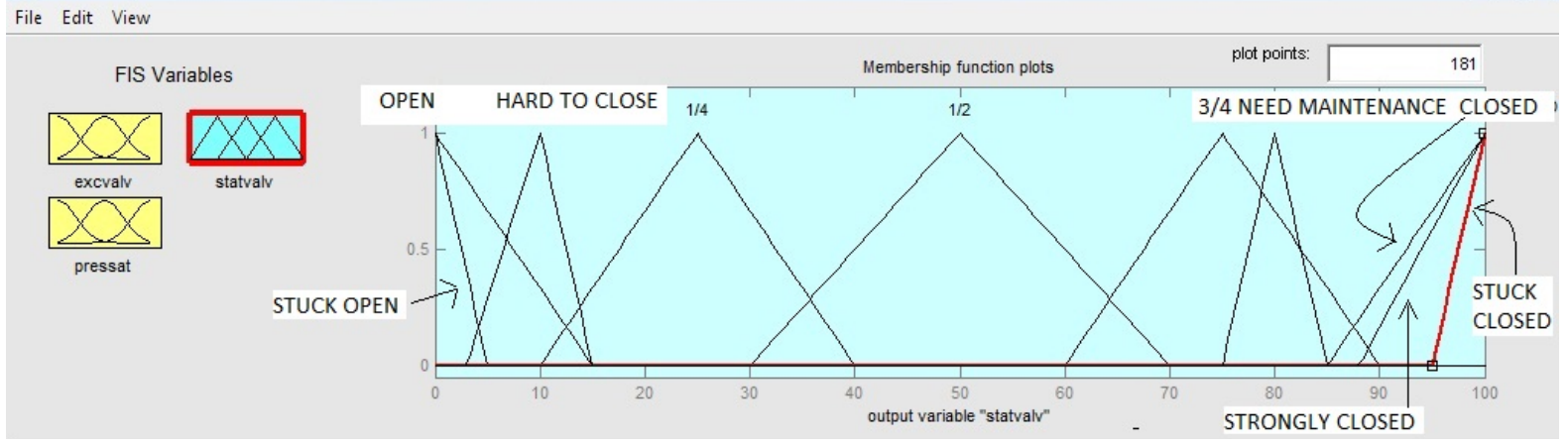

Figure 12. The output variable "statvalv".

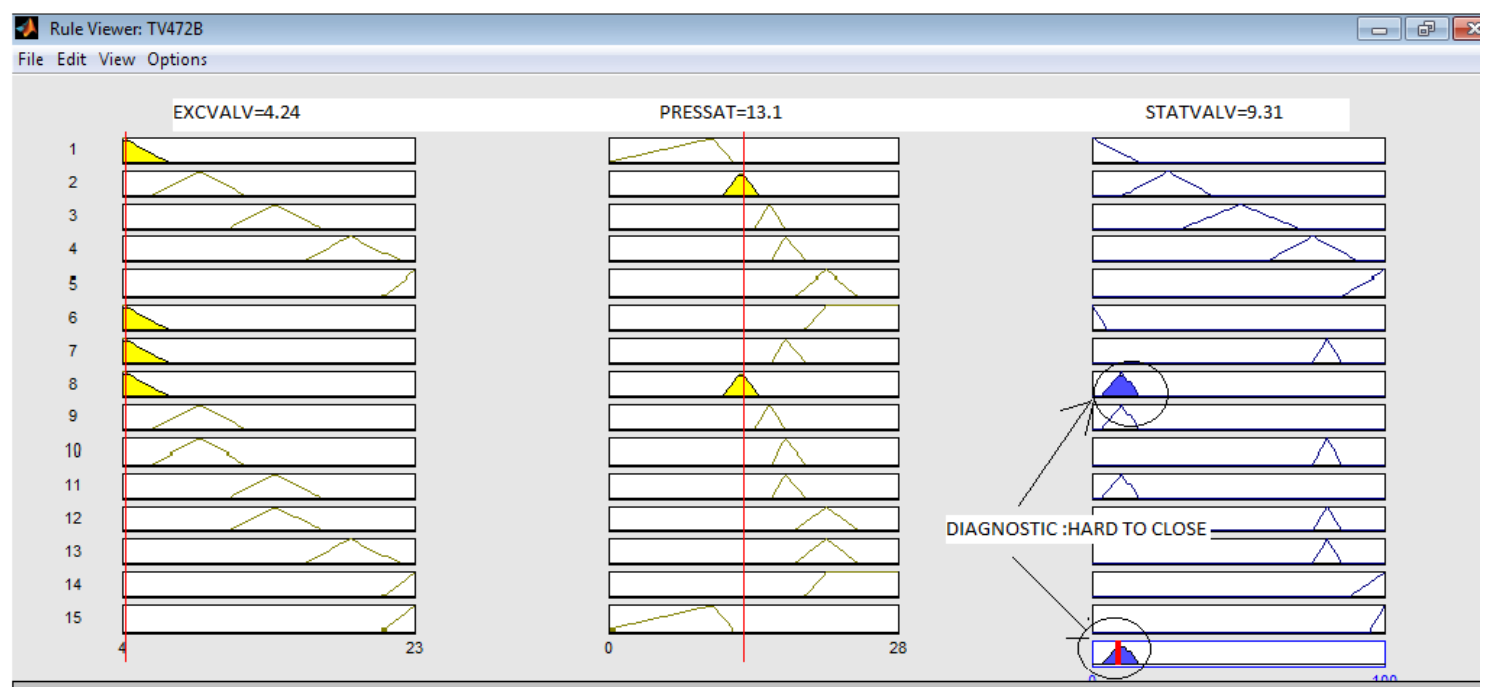

Figure 13. Rules viewer.

Table 4. The rules base.

RULES

1. If (excvalv is open) and (pressat is open) then (statvalv is open)

2. If (excvalv is a room) and (pressat is a room) then (statvalv is a room)

3. If (excvalv is in the middle) and (pressat is in the middle) then (statvalv is in the middle)

4. If (excvalv is three quarters) and (pressat is three quarters) then (statvalv is three rooms)

5. If (excralv is closed) and (pressat is closed) then (statvalv is closed)

6. If (excralv is open) and (pressat is strongly closed) then (statvalv is stuck open)

7. If (excvalv is open) and (pressat is three quarters) then (statvalv is set)

8. If (excvalv is open) and (pressat is a room) then (statvalv is difficult to close)

9. If (excvalv is a room) and (pressat is in the middle) then (statvalv is difficult to close)

10. If (excvalv is a room) and (pressat is three quarters) then (statvalv is necessary)

11. If (excvalv is in the middle) and (pressat is three quarters) then (statvalv is difficult to close)

12. If (excvalv is in the middle) and (pressat is closed) then (statvalv is in need of maintenance)

13. If (excvalv is three quarters) and (pressat is closed) then (statvalv is needed)

14. If (excralv is closed) and (pressat is strongly closed) then (statvalv is fort)

15. If (excvalv is closed) and (pressat is open) then (statvalv is stuck closed)

formance of the expert system mainly for failures on incipient stage. Furthermore the system must also be able to analyze different types of valves.

\section{References}

[1] Coble, J.B. and Upadhyaya, B.R. (2012) Prognostic and Health Management in Nuclear Power Plants: A Review of 
Technologies and Applications. National Technical Information Service.

[2] Guimarães Carneiro, A.L. (2003) Development of an Integrated Condition Monitoring and Diagnostic System for Motor-Operated Valves used in Nuclear Power Plant. Ph.D. Thesis, IPEN-USP, São Paulo, 22710. (in Portuguese).

[3] Pinto, C. and M. de Almeida, G. (2010) Failure Detection in a Control Final Element via Markov Model. Regional Meeting of Computational Applied Mathmatics_ERMAC, UFSJ, Ouro Branco. (in Portuguese).

[4] Guimarães Carneiro, A.L. and Porto Jr., A.C.S. (2013) Development of an Integrated Condition Monitoring and Diagnostic System for Process Control Valves Used in Nuclear Power Plant. AIDIC PHM, Milano, 872.

[5] Attaway, S. (2009) MATLAB ${ }^{\circledR}$ A Practical Introduction to Programming and Problem Solving. Elsevier, Amsterdam.

[6] Zadeh, L.A. (1984) Coping with the Imprecision of the Real World: An Interview with Lofti Zadeh. Communications of the ACM, 27, 304-311. http://dx.doi.org/10.1145/358027.358032

[7] Mendel, J.M. (1995) Fuzzy Logic Systems for Engineering: A Tutorial. Proceedings of the IEEE, 83, 83.

[8] Zadeh, L.A. (1965) Fuzzy Sets. Information and Control, 8, 338-353.

[9] Ross, T.J. (2010) Fuzzy Logic with Engineering Applications. 3rd Edition, Wiley, Hoboken. http://dx.doi.org/10.1002/9781119994374

[10] Mamdani, E.H. (1974) Application of Fuzzy Logic to Approximate Reasoning using Linguistic Synthesis. Department of Electrical and Electronic Engineering, Queen Mary College (University of London), London. 
Scientific Research Publishing (SCIRP) is one of the largest Open Access journal publishers. It is currently publishing more than 200 open access, online, peer-reviewed journals covering a wide range of academic disciplines. SCIRP serves the worldwide academic communities and contributes to the progress and application of science with its publication.

Other selected journals from SCIRP are listed as below. Submit your manuscript to us via either submit@scirp.org or Online Submission Portal.
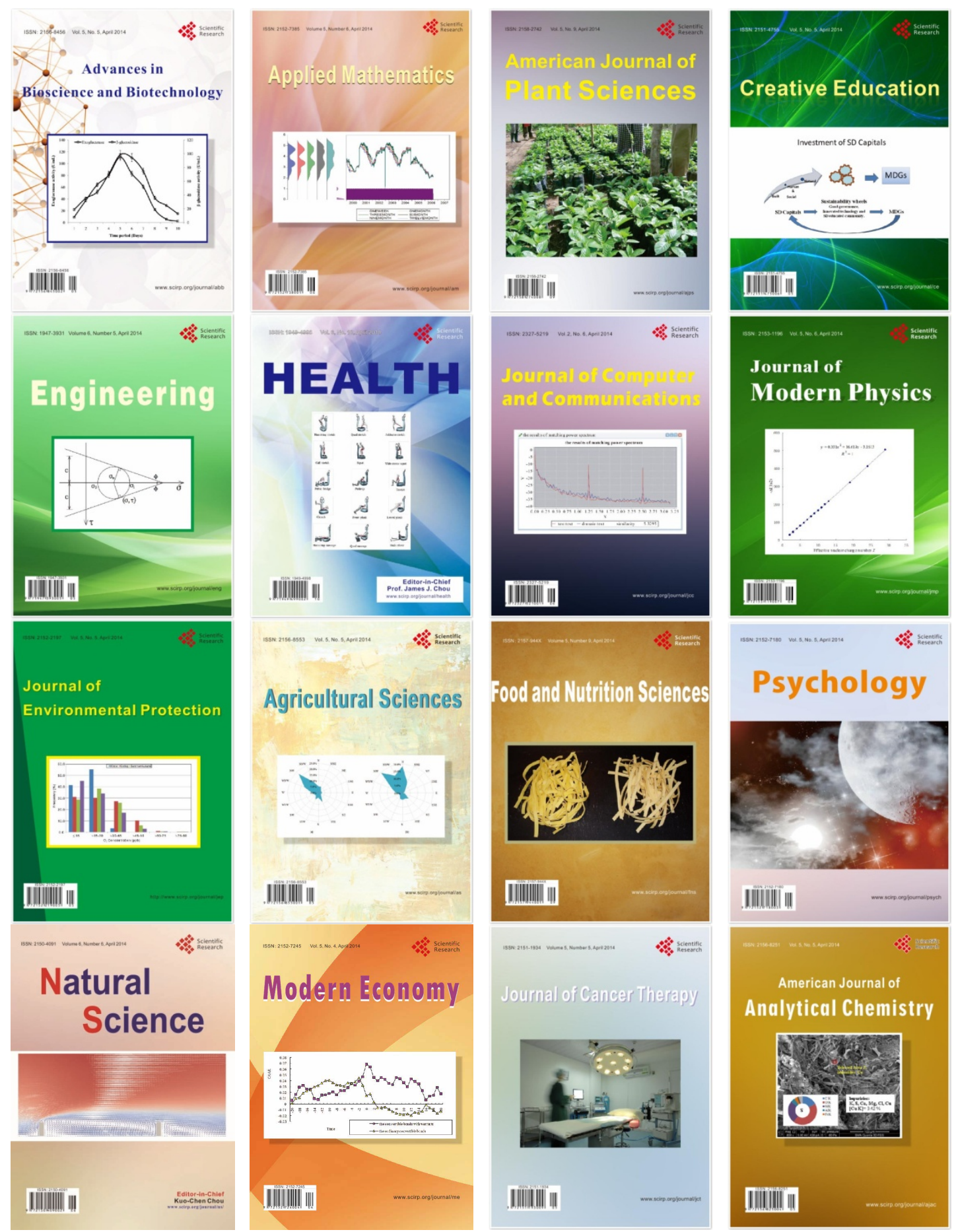